\title{
Quantitative Analysis of Magnetoferritin-Induced Relaxivity Enhancement in MRI
}

\author{
O. Strbak $^{a, *}$, L. Balejcikova ${ }^{b}$, M. Mihalikova ${ }^{c}$, P. Kopcansky ${ }^{d}$ And D. Dobrota ${ }^{c}$ \\ ${ }^{a}$ Biomedical Center Martin, Jessenius Faculty of Medicine in Martin, Comenius University in Bratislava, \\ Mala Hora 4, 03601 Martin, Slovakia \\ ${ }^{b}$ Institute of Hydrology, Slovak Academy of Sciences, Dubravska cesta 9, 84104 Bratislava, Slovakia \\ ${ }^{c}$ Department of Medical Biochemistry, Jessenius Faculty of Medicine in Martin, Comenius University in Bratislava, \\ Mala Hora 4, 03601 Martin, Slovakia \\ ${ }^{d}$ Institute of Experimental Physics, Slovak Academy of Sciences, Watsonova 47, 04001 Kosice, Slovakia

\begin{abstract}
Various disorders, including neurodegenerative diseases, are associated with iron accumulation. Ferritin particles are believed to be a precursor of pathological iron core transformation and iron accumulation, forming so-called pathological ferritin. Magnetoferritin is currently considered as the most suitable model system of pathological ferritin. It is described as apoferritin containing a magnetite nanocrystal. The magnetic moment of magnetoferritin is significantly larger than the magnetic moment of native ferritin containing ferrihydrite crystal. Theoretically, the effect of magnetoferritin on longitudinal and transverse relaxivity should also be significantly higher. Therefore, we provide a quantitative analysis of the magnetoferritin-induced enhancement of longitudinal and transverse relaxivity. However, our results at $7 \mathrm{~T}$ MRI indicate that a transverse relaxation significantly prevails in magnetoferritin compared to native ferritin. Such a quantitative analysis is essential for developing the MRI methodology required for non-invasive diagnostics of pathological processes associated with iron accumulation.
\end{abstract}

DOI: 10.12693/APhysPolA.137.720

PACS/topics: ferritin, magnetoferritin, MRI, relaxivity, non-invasive diagnostics

\section{Introduction}

Biological iron is associated with a variety of pathological processes, particularly neuroinflammatory processes [1], and neurodegenerative diseases [2]. It is generally accepted that these pathological processes are associated with disrupted iron homeostasis that results in iron accumulation and the formation of aggregates in the form of nanosized iron oxide particles [2]. Ferritin has been proposed as the precursor of such accumulated iron [3]. It is formed by a protein envelope $(12 \mathrm{~nm})$ and a mineral core $(2-7 \mathrm{~nm})$ in the form of crystalline ferrihydrite [4]. The primary role of ferritin in the body is the elimination of toxic ferrous ions upon reaching their critical concentration for the organism and depositing them in the form of nontoxic ferric ions for later usage by the organism. In 2004, Quintana et al. utilized electron nanodiffusion and electron microscopy to show that the ferritin mineral nucleus of patients with Alzheimer's disease (referred to as "pathological" ferritin) is structurally different from native (physiological) ferritin [3]. In physiological ferritin, the mineral core consists mainly of hexagonal ferrihydrite, hematite, and a smaller phase of magnetite. In contrast, the core of pathological ferritin consists mainly of cubic structures, such as magnetite and wüstite, and to a lesser extent ferrihydrite, with the absence of hematite. These

*corresponding author; e-mail: oliver.strbak@centrum.cz conclusions have been confirmed by a recent study [5], where muon spin rotation was used to show that the ferritin particles have a crystalline phase with large magnetocrystalline anisotropy that is compatible with magnetite or maghemite. Pathological ferritin is thus better described by magnetoferritin, which is composed of apoferritin and an artificially added phase of magnetite or maghemite [6]. The magnetic moment of magnetoferritin is significantly larger than the magnetic moment of the native ferritin core, therefore a more than 200 -fold increase in the longitudinal and transverse relaxation rates of magnetoferritin is expected [5]. Based on these theoretical expectations, we provide a quantitative analysis of the longitudinal and transverse relaxation rates, as well as the relaxivity, of magnetoferritin and native ferritin particles with a $7 \mathrm{~T}$ MRI system. These results should contribute to the development of an MRI methodology required for non-invasive diagnostics of pathological processes associated with iron accumulation (neurodegenerative disorders, neuroinflammation, cirrhosis, lung and heart diseases).

\section{Materials and methods}

Magnetoferritin was prepared by the incorporation of ferrous ions into the empty protein shell of native apoferritin by the synthesis method described in [7]. Three different loading factors, representing the average number of iron atoms per apoferritin, were prepared: $\mathrm{LF}_{\mathrm{A}}=553$, $\mathrm{LF}_{\mathrm{B}}=733$, and $\mathrm{LF}_{\mathrm{C}}=872$. The loading factor of native ferritin (NF) was determined as 884. Quantitative analysis of the loading factor was performed using a UV-vis 
spectrophotometer (SPECORD 40, Analytik Jena) at 2cc with a precision of $\approx 1 \%$.

The MRI relaxivity measurements were performed using a $7 \mathrm{~T}$ BioSpec Bruker system. The concentration gradient of iron oxide $\left(2.5 \times 10^{-3}-0.02\right) \mathrm{mg} / \mathrm{ml}$ of ferrihydrite in ferritin, and magnetite in magnetoferritin) and two different MRI protocols were used for the determination of the longitudinal and transverse relaxation rates $\left(R_{1}\right.$ and $\left.R_{2}\right)$ and relaxivity $\left(r_{1}\right.$ and $\left.r_{2}\right)$ :

- RARE - rapid acquisition with refocused echoes ( $T_{1}$ mapping),

- MSME - multi-slice multi-echo ( $T_{2}$ mapping).

The relaxation rate $R_{n}$ is inverse to the relaxation time

$$
R_{n}=\frac{1}{T_{n}}, \quad(n=1 \text {, or } 2) .
$$

The relaxation times were determined by fitting the signal intensity values. The change in $R_{n}$ is defined as the relaxivity of the magnetic particles:

$$
r_{n}=\frac{R_{n}-R_{n}^{0}}{C} \quad(n=1, \text { or } 2),
$$

where $R_{n}^{0}$ is the relaxation rate in the absence of iron oxide core, $R_{n}$ represents the relaxation rate in the presence of iron oxide core, and $C$ is the iron oxide concentration.

The Paravision Image Sequence Analysis tool (Bruker, Germany), and Matlab R2019a (Mathworks Inc., USA) were employed for data processing.

\section{Results}

Comparisons of the longitudinal and transverse relaxation rates of native ferritin and magnetoferritin are shown in Fig. 1a and b, respectively. The relaxation rates were calculated from Eq. (1), while the relaxation times were determined by fitting the signal intensity. For both relaxation rates, all the magnetoferritins with different loading factors are clearly distinguishable from the native ferritin. However, for the longitudinal relaxation rate $R_{1}$ (Fig. 1a), the difference is not so significant as the contrast in the transverse relaxation rate $R_{2}$ (Fig. 1b). Quantitatively, these differences are shown in Fig. $1 \mathrm{c}$ and $1 \mathrm{~d}$, as the ratio of magnetoferritin to native ferritin. While for the longitudinal relaxation rate $R_{1}$, the range is from $\approx 1.1$ to 1.8 in favor of magnetoferritin, for the transverse relaxation rate $R_{2}$, it is already up to $\approx 7-50$ in account of magnetoferritin.

The longitudinal and transverse relaxivity of native ferritin and magnetoferritin are described in Figs. 2a and $2 \mathrm{~b}$. Similar to the relaxation rate changes, the longitudinal relaxivity (Fig. 2a) of magnetoferritin is only slightly higher $\left(r_{1}\left(\mathrm{LF}_{\mathrm{A}}\right)=2.17 \mathrm{mM}^{-1} \mathrm{~s}^{-1}, r_{1}\left(\mathrm{LF}_{\mathrm{B}}\right)=\right.$ $1.5 \mathrm{mM}^{-1} \mathrm{~s}^{-1}$, and $\left.r_{1}\left(\mathrm{LF}_{\mathrm{C}}\right)=1.2 \mathrm{mM}^{-1} \mathrm{~s}^{-1}\right)$ in comparison with native ferritin, namely $r_{1}(\mathrm{NF})=0.4 \mathrm{mM}^{-1} \mathrm{~s}^{-1}$. In contrast, the transverse relaxivity (see Fig. 2b) of magnetoferritin $\left(r_{2}\left(\mathrm{LF}_{\mathrm{A}}\right)=320 \mathrm{mM}^{-1} \mathrm{~s}^{-1}, r_{2}\left(\mathrm{LF}_{\mathrm{B}}\right)=\right.$ $527.7 \mathrm{mM}^{-1} \mathrm{~s}^{-1}$ and $\left.r_{2}\left(\mathrm{LF}_{\mathrm{C}}\right)=402.3 \mathrm{mM}^{-1} \mathrm{~s}^{-1}\right)$
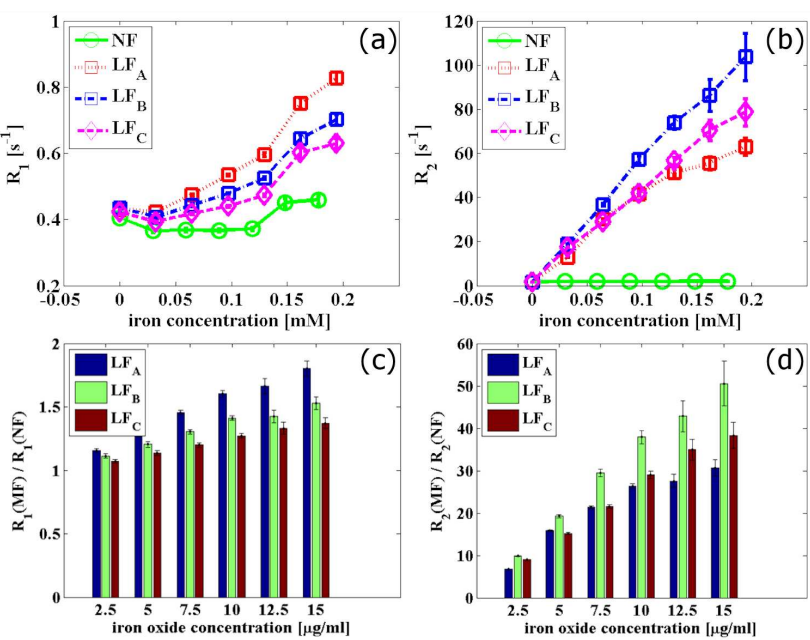

Fig. 1. Comparison of native ferritin and magnetoferritin: (a) longitudinal relaxation rate $R_{1}$, (b) transversal relaxation rate $R_{2}$. Ratio of magnetoferritin to native ferritin: (c) longitudinal relaxation rate $R_{1}$, (d) transversal relaxation rate $R_{2}$.
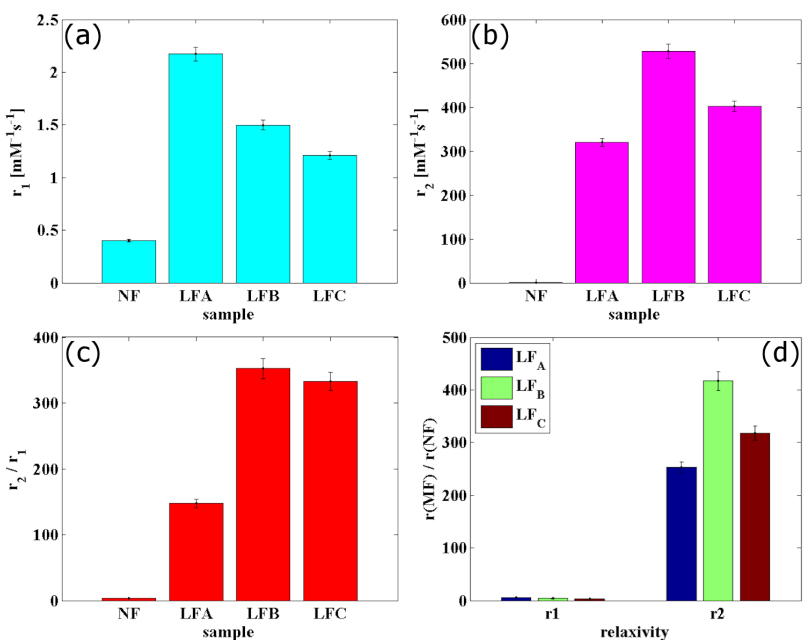

Fig. 2. Relaxivity comparison of native ferritin and magnetoferritin: (a) longitudinal relaxivity $r_{1}$, (b) transverse relaxivity $r_{2}$, (c) ratio of transverse and longitudinal relaxivity $r_{2} / r_{1}$, (d) relaxivity ratio of magnetoferritin to native ferritin.

is significantly larger in comparison with native ferritin $\left(r_{2}(\mathrm{NF})=1.27 \mathrm{mM}^{-1} \mathrm{~s}^{-1}\right)$. The ratio of transverse and longitudinal relaxivity $\left(r_{2} / r_{1}\right)$ is high (Fig. 2c), proving the characteristics of magnetoferritin as a $T_{2}$ contrast agent [8]. The quantitative analysis of the magnetoferritin-induced relaxivity enhancement is displayed in Fig. 2d. The ratio of magnetoferritin and native ferritin is of the order of less than 10-times for longitudinal relaxivity $\left(\mathrm{LF}_{\mathrm{A}}=5.4, \mathrm{LF}_{\mathrm{B}}=3.7\right.$, and $\mathrm{LF}_{\mathrm{C}}=3.0$ ), while for transverse relaxivity, it is of order of 100 -times $\left(\mathrm{LF}_{\mathrm{A}}=252.9, \mathrm{LF}_{\mathrm{B}}=416.9\right.$, and $\mathrm{LF}_{\mathrm{C}}=317.8$ ). 


\section{Discussion}

Based on theoretical calculations, a more than 200 times larger effect of magnetite-like particles (magnetoferritin) is expected on $R_{1}$ and $R_{2}$ of the surrounding water protons compared to the effect of ferrihydrite-like particles (native ferritin) [9]. However, our results indicate the minimal effect of synthetically prepared magnetoferritin on $R_{1}$ at $7 \mathrm{~T}$ (Fig. 1a). Depending on the loading factor and iron oxide concentration, it is in the range of 1.1 to 1.8 times (Fig. 1c). In contrast, $R_{2}$ is significantly larger in magnetoferritin than in native ferritin (Fig. 1b). Quantitatively, it ranges from an almost 10 times enhancement to a $\approx 50$ times enhancement (Fig. 1d). Although it is not the expected value, the comparison with [10] is very similar. At $7 \mathrm{~T}$, they observed a 125 -fold increase in the transverse relaxation rate of magnetoferritin in comparison with normal brain ferritin. However, the result was normalized for an iron concentration of $10 \mathrm{mM}$, which is still significantly more than our most concentrated sample ( $0.24 \mathrm{mM}$ of iron). The $r_{1}$ and $r_{2}$ values naturally copy the values of the relaxation rates (Fig. 2a and $2 \mathrm{~b}$ ), indicating the prevailing transverse relaxation effect of magnetoferritin on surrounding water protons. This is also supported by the high $r_{2} / r_{1}$ ratio of magnetoferritin (from 147.3 to $352.2,3.2$ for native ferritin, Fig. 2c) that characterizes the efficiency of the paramagnetic molecule for contrast properties [7]. The observed transverse relaxivity of magnetoferritin is even larger compared to commercially used iron oxide contrast agents: Feridex, $r_{2}=120 \mathrm{mM}^{-1} \mathrm{~s}^{-1}$; Resovist, $r_{2}=186 \mathrm{mM}^{-1} \mathrm{~s}^{-1}$; Combidex, $r_{2}=65 \mathrm{mM}^{-1} \mathrm{~s}^{-1}$ [7]. The ratio of magnetoferritin relaxivity to native ferritin relaxivity in Fig. 2d quantitatively proves the significant effect of transverse relaxation (252.9-416.9 times enhancement) in comparison with longitudinal relaxation (3.0-5.4 times enhancement).

\section{Conclusions}

We have quantified the effect of magnetoferritin nanoparticles on longitudinal and transverse relaxation rates and relaxivity in comparison with native ferritin. Our results show the prevailing effect of magnetoferritin particles on the transversal relaxation of surrounding water protons at 7 T. Such a quantitative analysis is required for the development of an MRI methodology for the non-invasive diagnostics of pathological processes associated with iron accumulation (neurodegenerative disorders, neuroinflammation, cirrhosis, lung and heart diseases).

\section{Acknowledgments}

This work was supported by the projects: Ministry of Health (2018/11-UKMT-7), VEGA (2/0016/17, 2/0044/20), APVV (APVV-015-0453), and Competence Center Martin (ITMS code: 26220220153).

\section{References}

[1] T.C. Frank-Cannon, L.T. Alto, F.E. McAlpine, M.G. Tansey, Mol. Neurodegener. 16, 47 (2009).

[2] J. Hagemeier, J.J.G. Geurts, R. Zivadinov, Expert Rev. Neurother. 12, 1467 (2012).

[3] C. Quintana, J.M. Cowley, C. Marhic, J. Struct. Biol. 147, 166 (2004).

[4] N.D. Chasteen, P.M. Harrison, J. Struct. Biol. 126 , 182 (1999).

[5] L. Bossoni, L.G. Moursel, M. Bulk, et al., J. Phys. Condens. Matter 29, 415801 (2017).

[6] L. Xue, D. Deng, J. Sun, Int. J. Mol. Sci. 20, 2426 (2019).

[7] O. Strbak, L. Balejcikova, L. Baciak, J. Kovac M. Masarova-Kozelova, A. Krafcik, D. Dobrota, P. Kopcansky, J. Phys. D Appl. Phys. 50, 365401 (2017).

[8] J. Estelrich, M.J. Sanchez-Martin, M.A. Busquets, Int. J. Nanomed. 10, 1727 (2015).

[9] A. Roch, R.N. Muller, P. Gillis, J. Chem. Phys. 110, 5403 (1993).

[10] Y. Gossuin, D. Hautot, R.N. Muller, Q. Pankhurst, J. Dobson, C. Morris, P. Gillis, J. Collingwood, NMR Biomed. 18, 469 (2005). 